\title{
Novel clumped isotope constraints on terrestrial hydroclimates of Western North America during the Last Glacial Maximum and Deglaciation
}

\author{
ARADHNA TRIPATI ${ }^{1}$ AND ALEXANDREA ARNOLD ${ }^{2}$ \\ ${ }^{1}$ UCLA \\ ${ }^{2}$ University of California - Los Angeles \\ Presenting Author: atripati@g.ucla.edu
}

The "wet gets wetter, dry gets drier" scenario has been a common school of thought in the climatological community, with warming associated with an overall intensification of the hydrologic cycle. However, this simplified scenario of the hydrologic cycle is derived primarily from theory and oceanic data, and modeling studies that suggest that it may not reflect the intricacies of the hydrologic cycle on land. Over large parts of North America, model simulations disagree over whether past and future warming is associated with more or less rainfall in certain regions. This disagreement arises from the complex interplay between atmospheric, oceanic, and terrestrial processes and their response to rising greenhouse gases, combined with differences in the depictions of these processes in simulations. Quantitative terrestrial reconstructions of hydroclimate parameters can provide critical benchmarks to evaluate models, but are relatively sparse. Here, we present a novel thermodynamically-based approach that uses carbonate clumped isotope measurements of paleosol and lacustrine carbonates to quantify terrestrial temperatures, precipitation rates, evaporation rates, and water isotopes. We apply this tool to Last Glacial Maximum and deglacial carbonates from several basins in Western North America, and evaluate model skill. Case studies will be used to show how we can examine the relative role of evaporation and precipitation in local hydrologic budgets, identify whether summer or winter precipitation was dominant, and explore what source regions and transport pathways for vapor were. These data are used to identify broad-scale dynamical mechanisms that control spatial and temporal changes in the water cycle, to test hypotheses about the role of thermodynamic and dynamic drivers of changes in the moisture balance in the US and Mexico, including shifts in the westerlies and changes in the North American monsoon. 